\title{
Développement de la créativité collaborative par la pratique du Hackathon. Effet de levier pour le développement des bases des futures innovations
}

\author{
Development of collaborative creativity through the practice of the \\ Hackathon. Leverage effect for the development of the basis of future
} innovations

\author{
Dave Mobhe Bokoko ${ }^{1}$ \\ ${ }^{1}$ Laboratoire de Recherche sur l'Industrie et l'Innovation, Université du Littoral Côte d'Opale, \\ mobheabdave@gmail.com
}

RÉSUMÉ. La propagation d'internet et l'avènement d'une ère digitale ont brutalement rendu disponibles des stocks considérables de connaissances tout en accélérant leur diffusion. Ce contexte particulier invite les entreprises à développer des organisations agiles et collaboratives capables d'associer l'écosystème afin d'innover plus vite, plus souvent...bref, innover de manière créative. La tendance actuelle montre que les entreprises sont de plus en plus dans une démarche ouverte et collaborative, s'appuyant généralement sur la promotion de la créativité, notamment par l'adoption des pratiques comme le hackathon. La question à laquelle nous allons répondre dans cet article est de savoir comment les hackathons par le levier de la créativité collaborative posent les bases des futures innovations. Cette question se justifie par la capacité du hackathon à s'appuyer sur la créativité et la collaboration avec l'écosystème dans une démarche co-créative et agile afin de développer des nouvelles hypothèses qui serviront de bases pour les futures innovations.

ABSTRACT. The spread of the Internet and the advent of a digital age have brutally made available vast stocks of knowledge while accelerating their diffusion. This context invites companies to develop agile and collaborative organizations capable of associating the ecosystem in order to innovate faster, more often ... in short, to innovate creatively. The current trend shows that companies are increasingly taking an open and collaborative approach, generally based on the promotion of creativity, including through the adoption of practices such as the hackathon. The question we will answer in this article is how by leveraging collaborative creativity hackathons lay the foundations for future innovations. This question is justified by the hackathon's ability to rely on creativity and collaboration with the ecosystem in a co-creative and agile approach in order to develop new hypotheses that will serve as the basis for future innovations.

MOTS-CLÉS. Hackathon, Créativité collaborative, Innovation, Design Thinking, Lean Startup.

KEYWORDS. Hackathon, Collaborative creativity, Innovation, Design Thinking, Lean Startup.

\section{Introduction}

La propagation d'internet et l'avènement d'une ère digitale ont brutalement rendu disponibles des stocks considérables de connaissances tout en accélérant leur diffusion. Dans ce contexte particulier, les entreprises les plus innovantes ont cessé de concevoir leur processus d'innovation comme un domaine stratégique, réservé exclusivement aux chercheurs, ingénieurs et marketeurs ${ }^{1}$. En effet, pour accélérer la dynamique de croissance et rester compétitives, les entreprises font de plus en plus appel à tout leur écosystème dans une logique d'Open innovation (Chesbrough, 2006) - par le biais $\mathrm{d}^{\prime}$ innovation participative ${ }^{2}$, de crowdsourcing, de marketing collaboratif ${ }^{3}$, etc. - afin de soutenir un flux important d'innovations.

\footnotetext{
${ }^{1}$ Francisation de l'anglais marketer, le terme fait référence au spécialiste du marketing.

${ }^{2}$ Consiste à s'appuyer sur la capacité d'innovation de tous les collaborateurs (Fernez-Walch \& Romon, 2010, p.250).

${ }^{3}$ Consiste à associer des utilisateurs pilotes au processus d'émergence des projets d'innovation (Ibid., p.252).

(c) 2020 ISTE OpenScience - Published by ISTE Ltd. London, UK - openscience.fr 
L'impérative d'innover passe donc par une approche créative combinant différents facteurs de production de sources diverses, tant internes qu'externes. Le point d'impulsion de la capacité à innover devient dès lors celle de la mobilisation du capital humain dans sa capacité à faire différentes associations afin de générer des solutions inédites à des problématiques latentes ou non résolues. De ce fait, les entreprises, s'appuyant sur le potentiel créatif comme levier du processus de création de valeur, seraient plus enclines à rendre encore plus performants les modèles économiques existants et/ou d'en créer des nouveaux. Un peu comme pour reprendre Albert Einstein : « l'imagination est plus importante que le savoir ». Avec cette prémisse, la créativité pourrait se définir comme un processus mental individuel impliquant la production de nouvelles idées ou concepts nouveaux [LAM 17, P.9]. Par conséquent, jouant le rôle d'ouverture et d'exploration des nouvelles possibilités, la créativité s'avèrerait être un levier efficace de production d'innovations.

Par ailleurs, nous assistons à un véritable engouement mondial pour les pratiques d'innovations ouvertes et participatives. Il est important de noter que cela est dû à leur capacité à stimuler la créativité et l'innovation au sein des entreprises. Bien loin des boites à idées et des cercles de qualité, plusieurs de ces pratiques d'entreprises prennent forme d'événements collaboratifs d'innovation [STER 95] tels que les ateliers de créativité ou d'innovation, les Design sprint, les concours d'innovation en ligne, les Startup Weekend, les hackathons, etc. Selon BemyApp [BEM 19], on enregistre une nette évolution de 40\% de 2016 à 2018 en nombre de hackathons. Ces pratiques se présentent sous forme de concours d'innovation allant généralement de 12 à 72 heures. En effet, ces pratiques dites «innovantes » ont pour objectif de promouvoir la créativité - plus précisément la créativité collaborative - en s'appuyant sur les interactions et collaboration entre acteurs divers pour générer le maximum d'idées et solutions nouvelles, et ce afin d'accélérer le développement d'un grand nombre de projets d'innovations dans des délais impartis.

De ce qui précède, nous constaterons que la créativité et l'innovation sont ipso facto des éléments vitaux pour développer des modèles économiques encore plus performants. Autrement dit, les pratiques d'innovation susmentionnées s'appuient sur les outputs issus de la collaboration entre différents acteurs d'un écosystème d'innovation à la suite d'un degré plus important d'utilisation du capital humain - par le levier de la créativité - in fine pour développer le plus de projets d'innovation, et ce afin de rester compétitif. Tout cela nous conduit donc à analyser : « comment les hackathons par le levier de la créativité collaborative développent les bases des futures innovations pour les entreprises? »

Nous noterons que dans la pratique courante les notions sur la créativité et l'innovation semblent être interchangeables. Certains projets créatifs sont souvent automatiquement considérés comme innovants. De ce fait, pourrait-on avoir le même reflexe pour les projets innovants en tant que projets créatifs ? D'où la nécessité de dégager le lien pouvant exister entre la créativité et l'innovation dans un premier lieu ; ensuite, nous analyserons dans quelle mesure la pratique du hackathon s'appuie sur la créativité et la fédère pour accélérer le processus d'innovation des entreprises.

\section{La créativité au service de l'innovation}

L'histoire nous démontre que tous les grands innovateurs à l'instar de Nikola Tesla, Thomas Edison, Steve Jobs, Bill Gates, Mark Zuckerberg, etc. étaient ou sont tous des êtres très créatifs. De ce fait, pouvons-nous pleinement affirmer que les individus créatifs sont innovants par nature ? La créativité est-elle liée à des aspects génétiques des individus ? En effet, ces quelques interrogations soulèvent encore plus la pertinence de ce thème tout en générant autant de questions d'ordre conceptuel que pratique, à savoir : Qu'est-ce que la créativité ? Qu'est-ce qu'est l'innovation ? Existe-t-il un lien entre la créativité et l'innovation? 


\subsection{De la créativité à l'innovation}

\subsubsection{Le processus créatif}

Le concept grec de la créativité nous renvoie à 500 ans av. J.-C, où les grecs croyaient qu'ils vivaient dans un monde où l'originalité était uniquement donnée par les dieux. De même pour Socrate, les pensées et idées étaient toutes d'inspiration divine. Dans cette conception, la créativité ne pouvait guère provenir d'une personne rationnelle. Au début du XIXe, la créativité commença à être considérée comme provenant d'une partie secrète du cerveau. Mais là encore, il s'agit d'une conception de plus cantonnant la créativité à une dimension élitiste.

A travers la littérature que ce soit du domaine de la psychologie, la sociologie, l'économie et de la gestion, on peut observer une évolution historique dans les concepts de créativité. De fait, la définition de la créativité reste un sujet de recherche en soi et pour lequel les débats scientifiques sont toujours d'actualité. Il existe un bon nombre de définitions admises par la plupart des chercheurs. Nous définirons la créativité comme « la capacité à réaliser une production qui soit à la fois nouvelle et adaptée au contexte dans lequel elle se manifeste » [MAC 62] [BAR 88] [OCH 90] [LUB 94] [STER 95] [AMA 96] [AMA 12] [AMA 02] [RUN 12] [AND 14] [DES 08] cité par [LUB 15]. Selon De Bono [DEB 92], la créativité fait référence à la capacité de faire des nouvelles associations d'idées en vue de générer des nouveaux concepts. Au sens plein, l'acte créatif implique à la fois la génération d'idées et la manifestation de celles-ci afin qu'il se produise un résultat [SAM 07]. Dès lors, la créativité comprend la notion «d'application », caractéristique qui la rend aussi exploitable dans le monde des entreprises lorsqu'elle apparait sous la forme de capacité à développer des solutions aux problèmes identifiés ou non. Conséquemment, la définition retenue par notre étude se veut démêler toute confusion éventuelle qui pourrait se créer entre la créativité artistique - domaine de la beauté et du fantastique, qui d'ailleurs n'est pas toujours créative - et la capacité à changer de perception, à créer des concepts nouveaux.

Nous devons donc intégrer que la créativité n'est pas qu'un simple résultat - telle une manne tombée du ciel. Bien au contraire, elle découle d'un processus qui peut être observé et décrit. Tout au long de notre étude, nous parlerons délibérément de «processus créatif », afin de prendre de la distance par rapport à une perception de folie souvent accordée au concept de créativité. Dans la littérature, il existe plusieurs modélisations du processus créatif qui, traditionnellement, reprennent 4 phases telles que la préparation, l'exploration, l'incubation ${ }^{4}$, et enfin la vérification [WAL 26] ${ }^{5}$ [BOU 04] [BES 15] [LUB 15].

Tout au long de ce processus créatif, on peut distinguer deux types de séquence qui se succèdent : d'une part la « pensée divergente » qui est la capacité intellectuelle à produire de nombreuses idées, elle part de la phase préparatoire à l'exploration ; et d'autre part, la « pensée convergente » qui est la capacité intellectuelle à sélectionner, associer, combiner et synthétiser différents éléments en vue de développer un concept amélioré ou nouveau, elle correspond à la phase d'incubation. Pour Besançon et Lubart [BES 15], ces deux types de pensées sont nécessaires pour la pleine expression du potentiel créatif.

Moreno (1939) ${ }^{6}$ insiste également sur la créativité comme une capacité qui peut être développée et orientée. Ainsi, la créativité peut alors être stimulée par des outils et méthodes. Les premières techniques formalisées de stimulation de la créativité au sein des organisations sont les célèbres

\footnotetext{
${ }^{4}$ Fait référence à la période de développement de l'embryon d'un œuf.

${ }^{5}$ Graham Wallas définit le mieux le modèle du processus créatif dans The Art of Thought (1926). Aujourd'hui, sa vision reste une référence.

${ }^{6}$ Cité par Agogué, M. (2016).

(c) 2020 ISTE OpenScience - Published by ISTE Ltd. London, UK - openscience.fr 
séances de Brainstorming mises au point par Osborn ${ }^{7}$ dans les années 1940. En 1946, le TRIZ ou « Théorie de résolution des problèmes inventifs » est développé par l'ingénieur soviétique Genrich Altshuller - en s'appuyant sur la stimulation de la créativité - pour parvenir à débloquer l'inertie mentale des ingénieurs. Plus tard dans les années soixante, le concept de «la pensée latérale » est développé par Edward de Bono dans son livre The Use of Lateral Thinking (1967). La pensée latérale est associée à plusieurs techniques de créativité, elle a servi au développement de plusieurs outils et méthodes de créativité.

La créativité fait l'objet d'un nombre croissant de recherches. En effet, elle joue un rôle central dans l'ouverture et l'exploration du champ des possibilités dans le développement des nouveaux concepts. Cette section ne fera point l'inventaire de toutes les techniques de créativité existantes. Néanmoins, la multitude croissante des méthodes développées pour stimuler la créativité en toutes en commun la mise en œuvre d'une étape rationnelle permettant de classer, sélectionner et développer les meilleures solutions via l'expérimentation quel que soit le domaine d'activité.

\subsubsection{Le fait innovant}

L'innovation est au cœur de la dynamique économique. Mais de quoi parle-t-on exactement lorsqu'on parle d'innovation? Schumpeter a été le premier à noter et, surtout à mettre en avant les différences entre innovation et invention [CAR 07] [PEN 16] [COH 16]. L'invention est une nouvelle solution technique à un problème concret. L'innovation quant à elle consiste à introduire l'invention dans le circuit économique, i.e. à lui donner de la valeur économique. L'invention ne se transforme qu'en innovation que lorsqu'elle a été introduite dans le circuit économique (par exemple lorsqu'elle est commercialisée pour une innovation de produit ou lorsqu'elle est utilisée par une entreprise pour innovation de procédé).

Longtemps considéré comme un simple résultat [FOR 14], la première modélisation de l'innovation fut celle du modèle linéaire et hiérarchique. Bien que ce modèle orientât fortement les premières politiques en matière de recherche et d'innovation dans les années 1950 et incita des nombreuses entreprises à augmenter leurs dépenses en Recherche \& Développement (R\&D) dans les années 1980 par la suite, il n'en reste pas moins qu'il fut marginalisé et contesté, car, rendant compte d'une approche réductionniste du processus d'innovation. Ce modèle imposait la recherche fondamentale comme étape pionnière de toute innovation [FOR 97]. Malheureusement, cette vision $\mathrm{R} \& \mathrm{D}$ ne prend pas en compte les diverses et complexes rétroactions qui viennent ponctuer le déroulement de tout processus d'innovation [HER 95].

Kline et Rosenberg [KLI 86] propose un nouveau modèle en chaine de liaison: le modèle d'innovation interactif. Ce modèle est dit interactif dans la mesure où il intègre - à l'inverse du modèle linéaire et hiérarchique - des diverses et complexes rétroactions et feedback qui, allant jusqu'à une interaction avec le marché, apportent une réactivité interdépendante et propice au développement des innovations. Par ailleurs, en plaçant la phase de conception au centre du processus, le modèle est le premier de son genre à souligner la dimension organisationnelle du processus d'innovation et « refuse toute puissance de la science sur le fait innovatif ${ }^{9}$ » [CHO 07].

Contrairement aux modèles classiques ${ }^{10}$ d'innovation, le modèle d'Open Innovation est basé sur l'idée selon laquelle il est nécessaire de dépasser les frontières des organisations pour survivre dans

\footnotetext{
${ }^{7}$ Alex Osborn, l'un des premiers théoriciens du raisonnement créatif, se distingue surtout par le développement de l'outil de résolution de problèmes créatifs le plus connu : « le brainstorming » dans Applied Imagination (1953).

${ }^{8}$ En intégrant le marché dans le processus d'innovation, la conception et la qualification des innovations se poursuivent jusque chez l'utilisateur (Mobhe Bokoko, 2019, Page 31).

${ }^{9}$ Fait référence à un ensemble d'actions et événements qui débouche à une innovation.

${ }^{10}$ Fait référence aux modèles linéaire et interactif.

(C) 2020 ISTE OpenScience - Published by ISTE Ltd. London, UK - openscience.fr 
un monde encore plus moderne et compétitif ${ }^{11}$ [CHE 06]. Popularisé en 2003 par Chesbrough, l'Open innovation trouve sa pertinence dans la conception d'un processus d'innovation avec des murs perméables afin que, dès les étapes amont d'un projet, des ouvertures et collaborations soient possibles avec tout l'écosystème.

Par ailleurs, dans une époque où presque tout est digitalisé, marquée par une grande et rapide diffusion de la connaissance, il serait presque utopique pour une entreprise d'envisager qu'elle détiendrait à elle seule, et pendant longtemps, les ressources et informations lui conférant un avantage concurrentiel permanent. C'est pourquoi l'ouverture et la coopération permettent à l'entreprise d'échanger et accéder à des ressources supplémentaires et complémentaires avec son écosystème, in fine avec pour conséquence le renforcement de sa capacité à innover.

\subsubsection{Lien entre le fait créatif et le fait innovant}

Soyez créatifs et innovez ! Ces injonctions jalonnent quotidiennement les discours des managers, des dirigeants et même des hommes politiques [COH 16]. Herman [HER 99] posait la question de savoir si la créativité et l'innovation sont différentes? Avons-nous besoin des deux ? ${ }^{12}$ Cela laisse penser qu'il existe des opinions diverses sur la créativité et l'innovation.

Avant de présenter le lien qui pourrait exister entre la créativité et l'innovation, les sections précédentes $(\$ 1.1 .1$ et $\S 1.1 .2)$ ont pu respectivement illustrer ces deux notions en tant que processus distincts. Pour la créativité, il s'agit d'un mécanisme intellectuel de génération de nouvelles idées, nouveaux concepts dans l'optique de résolution d'un problème, l'éloignant ainsi de son caractère élitiste. D'un autre côté, l'innovation représente le processus d'introduction dans le circuit économique d'une solution concrète nouvelle ou significativement améliorée, l'éloignant ainsi de l'invention. Ce bref rappel nous permet d'entrevoir leurs différences en premier lieu, et par la suite leur proximité en décrivant dans quelles conditions la créativité peut être un vecteur clé d'innovation.

L'innovation étant un vecteur de progrès des sociétés, l'observation faite de toutes ses modélisations révèle un point de départ commun qui sont « l'idée et la connaissance ${ }^{13}{ }$. De surcroit, l'idée et la connaissance sont considérées comme les déclencheurs de la phase de conception, ladite phase considérée comme le cœur de tout processus d'innovation depuis le modèle de Kline et Rosenberg [KLI 86]. En effet, des études ont démontré que les entreprises qui accordent une attention particulière à la conception ont cinq fois plus de chance d'innover que les autres et développent des innovations plus « novatrices » [IRI 07] [TET 09].

La créativité, pour sa part, se situe dès lors en amont de l'innovation telle que décrite ci-haut. Elle intervient au début d'un enchainement permettant la recherche de connaissances nécessaires pour la résolution d'une problématique définie dans un premier temps, et ensuite, s'achève par la génération des nouvelles idées qui serviront au développement des concepts nouveaux ou améliorés. De ce fait, l'innovation peut être considérée comme une spirale dont le point focal est la créativité ${ }^{14}$, et qui s'éloigne de plus en plus allant de la génération du concept nouveau jusqu'à son développement technique, c'est-à-dire l'invention. En somme, l'innovation étant la commercialisation de

\footnotetext{
${ }^{11}$ Fait référence à la mondialisation, à la levée des barrières physiques via le digital, à un nombre plus important de concurrents, au cycle de vie des produits qui se raccourcit de plus en plus, etc.

${ }^{12}$ Traduction française du titre de son livre " Creatitivity ? Innovation ? Are They Different ? Do We Need Both ?"

13 II s'agit de la connaissance issue de la recherche fondamentale pour le modèle linéaire ; des idées et connaissances provenant du marché potentiel pour le modèle interactif ; et enfin, des idées et connaissances comme principaux inputs du modèle d'Open innovation.

${ }^{14}$ Toute innovation ne résulte pas fatalement d'un effort de créativité, ce serait absurde de le prétendre, citons les cas de serendipité. Toutefois, la créativité s'avère être un facteur substantiel dans le développement des concepts nouveaux. (c) 2020 ISTE OpenScience - Published by ISTE Ltd. London, UK - openscience.fr Page | 5
} 
l'invention, certains pourraient décrire l'innovation comme la commercialisation de la créativité [GOL 17].

La créativité est partout présente et requise dans l'organisation [CAM 11]. Son effet dans le cadre de projets formels d'innovation est particulièrement évident, notamment en raison du développement des outils de créativité conçus pour parvenir à débloquer l'inertie mentale des acteurs pilotes du processus d'innovation (ingénieurs, marketeurs, chercheurs, etc.). De ce fait, la créativité joue un rôle important dans la construction des (nouvelles) hypothèses. Sans elle, nous ne pouvons utiliser que des concepts standards, en d'autres termes pas de possibilité d'innover !

\subsection{La créativité collaborative comme déclencheur d'innovation}

\subsubsection{De la coopération à l'innovation}

Dans les années 80, bien que dominées par un modèle de fermeture, Kline et Rosenberg [KLI 86] soulignaient que le bon fonctionnement ainsi que la bonne performance d'un processus d'innovation n'étaient pas exclusivement fonction du nombre de chercheurs engagés et des investissements consacrés à la R\&D. En effet, ils étaient également fonction des interactions entre les différents acteurs de ce processus, de l'efficacité de la coopération et de la façon avec laquelle est conduit la phase de conception.

Dans son ouvrage de 1996 The Death of Competition [MOO 96] et trois ans plus tôt dans un article devenu célèbre dans le Harvard Business Review intitulé Predators and Prey : A New Ecology of Competition [MOO 93], Moore livre sa vision de la « fabrique de l'innovation ${ }^{15}$ » et montre l'intérêt de la collaboration plutôt que d'être systématiquement focalisé sur la compétition et la concurrence, laissant entendre que les deux attitudes (compétition et coopération) peuvent être gérées de concert. Ainsi se développera la notion de «coopétition» née du mélange de la compétition et de coopération, le principe étant que l'on a plus à gagner à travailler avec des partenaires innovants - et ce, même s'il s'agit de concurrents - que contre eux.

Chesbrough [CHE 03] va plus loin dans son modèle d'Open innovation. L'auteur s'appuie sur la nécessité qu'ont les entreprises à ouvrir leur processus d'innovation, par le biais de collaboration en échangeant et associant leurs actifs (idées, connaissances, technologies, etc.) à ceux de leur écosystème externe afin de créer davantage de valeur.

En définitive, la coopération est une stratégie clé pour innover. Elle permet aux entreprises soit dans une approche «Innovation sortante » de valoriser les actifs peu ou non utilisées à destination de leur écosystème (i.e. création des spin-off ${ }^{16}$ pour l'exploration de nouveaux marchés), soit par une approche «Innovation entrante » d'acquérir des ressources extérieures pour enrichir leurs capacités à innover.

\subsubsection{De la créativité individuelle à la créativité collaborative}

Dans les sections précédentes, la créativité a été fondamentalement abordée selon un point de vue individuel, c'est-à-dire en tant que processus mental de génération d'idées et concepts. Cette approche limite la créativité en tant que compétence individuelle qui, introduit dans le processus organisationnel, pourrait être exploitée dans la production des concepts nouveaux pouvant soutenir une dynamique d'innovation et de croissance.

\footnotetext{
${ }^{15}$ Fait référence à un processus d'innovation performant.

${ }^{16}$ La spin-off consiste à créer une nouvelle société totalement indépendante à partir d'une branche d'activité existant préalablement au sein d'une firme. 
Depuis la propagation d'internet dans les années 90 et la vague de digitalisation qui s'en est suivie, les modes de collaboration au sein des entreprises se sont brutalement renforcés [MOB 19]. En ce sens, les services et départements de production des entreprises ont cessé d'être considérés comme des silos indépendants, pour se transformer en activités menées par des acteurs liés et impliqués. L'ouverture à la collaboration a ipso facto contribué à l'intensification des échanges entre les différents individus au sein d'une organisation.

A l'image de la réaction en chaine des réacteurs nucléaires, où chaque neutron en cassant un atome d'uranium libère au moins deux autres neutrons et provoque un dégagement de chaleur, chaque solution nouvelle (idée ou concept) produite par un collaborateur est l'opportunité pour l'ensemble des collaborateurs de rebondir sur une ou plusieurs autres solutions originales grâce à l'émulation créée. Ainsi, on parlera davantage de créativité collaborative, celle-ci fait référence à la capacité d'une organisation à générer et accumuler des idées et concepts nouveaux par cet effet synergique.

La créativité collaborative, à l'inverse de la créativité individuelle qui est perçue comme un processus mental, constitue dès lors un processus organisationnel durant lequel la créativité d'un groupe d'individus suit des phases itératives et séquentielles, à la suite des échanges, de l'ouverture et l'usage de l'intelligence collective ${ }^{17}$. Autrement dit, la créativité collaborative consiste dès lors en un processus organisationnel visant à générer et surtout faire accepter des nouveautés (idées, concepts, connaissances) au sein d'une organisation. Ainsi, on comprend mieux le développement et l'utilisation des séances collectives de créativité.

\subsubsection{Effet de la créativité collaborative sur l'innovation}

Combien de fois n'a-t-on pas lu qu'innover est la seule option des entreprises pour rester compétitives. Cependant, il sied de noter que l'innovation porte plusieurs formes et implique des ressources de sources diverses. Le plus grand défi pour les entreprises serait alors de développer le potentiel créatif afin de soutenir et renforcer leur capacité à innover. La créativité collaborative, si elle est encouragée par l'organisation, possède la particularité de mener à la collaboration des individus appartenant (souvent) à des environnements complexes et quelques fois bien distincts dans l'objectif d'une plus grande contribution dans le processus d'innovation.

Par ailleurs, la créativité collaborative résulte d'un ensemble d'interrelations développées entre différentes personnes, au sein des équipes ou de toute l'organisation, mais également en termes de réseaux d'acteurs plus étendus (écosystème). Bien menée, la créativité collaborative peut conduire à des innovations compte tenu de sa capacité à créer, transformer et diffuser de nombreuses idées et concepts nouveaux. En revanche, mal gérée celle-ci peut tout aussi bien étouffer la créativité individuelle des collaborateurs et par conséquent celle de toute l'organisation [GOR 19].

En définitive, la créativité collaborative permet de renforcer le potentiel créatif de toute l'organisation par l'implication d'un plus grand nombre d'acteurs. En effet, la forte implication des équipes accroit la production du nombre de solutions nouvelles proposées, celles-ci après avoir été valorisées en véritables projets d'innovation pour l'entreprise, viennent comme un effet de boomerang renforcer cette même dynamique implicative d'innovation...pour résumer: le résultat d'un cercle vertueux !

\section{Hackathon et créativité collaborative}

Bien que l'innovation soit le cœur de la survie des entreprises, aucun ne pourrait affirmer qu'il existe d'approche miracle pour impulser cette dynamique. La créativité collaborative constitue un

\footnotetext{
${ }^{17}$ L'intelligence collective désigne la capacité d'une communauté à faire converger intelligence et connaissances pour avancer vers un but commun, pour faire face à une situation donnée présent ou à venir. 
avantage stratégique dans la mesure où elle enrichit le processus d'innovation par le levier de l'implication de tous les acteurs. En même temps, la tendance actuelle montre que les entreprises sont de plus en plus dans une démarche ouverte et collaborative, s'appuyant généralement sur la promotion de la créativité au sein des organisations, notamment par l'adoption des pratiques comme le hackathon. De la découle plusieurs interrogations, à savoir : Qu'est-ce qu'un hackathon ? En quoi est-ce un levier de créativité collaborative? d'innovation?

\subsection{Origine et émergence des Hackathons}

\subsubsection{Hackathon : la démocratisation d'une pratique informatique}

Le terme «hackathon » vient de la juxtaposition de deux mots : « hack » et « marathon » $[\mathrm{KOM}$ 15]. Le mot hack représente la recherche et l'exploitation de lignes de codes (logiciels), et non pas comme une référence à la cybercriminalité. En ce sens, le mot hack renvoie à la résolution créative de problèmes, à la conception, au prototypage et au challenge ; tandis que le mot marathon indique tout naturellement l'intensité de l'effort pendant un temps court.

Ensemble, le terme formé « hackathon » indique l'effort intense et concentré - comme dans un marathon - dans le but de trouver un concept nouveau impliquant le développement d'une solution concrète (prototype, logiciel, maquette, Proof of concept, etc.). En d'autres mots, les hackathons sont des événements dans lesquels des équipes se font concurrence dans un délai très court dans le but de développer la meilleure solution à la problématique du promoteur de l'événement [UFF 17].

Le terme fut entendu pour la première fois en 1999 aux USA lorsque John Gage, vice-président de Sun Microsystems, challengea les participants de la conférence JavaOne ${ }^{18}$ de coder un programme pour le nouveau Palm V (un des ancêtres du smartphone). Par la suite, l'équipe marketing de l'entreprise nomma l'évènement «Hackathon » et l'objectif des compétiteurs fut de développer une nouvelle fonctionnalité infrarouge pour l'appareil [BOC 17]. La même année, les créateurs du système d'exploitation OpenBSD utilisèrent le terme «Hackathon » lors d'un évènement à Calgary (CAN), rassemblant une dizaine de développeurs informatiques travaillant sur des systèmes de cryptographie.

Le pratique du hackathon se démocratisa dans les entreprises à partir des années 2000 [BOC 17], en grande partie, propulsé par la propagation d'internet et des technologies digitales. De plus, la pratique est perçue de plus en plus par les entreprises technologiques et non technologiques, ainsi que par les investisseurs en capital-risque comme un moyen de développer rapidement de nouvelles technologies logicielles, de prototyper et tester des solutions numériques en quelques jours ou quelques heures.

\subsubsection{Hackathon : une pratique émergente des entreprises innovantes}

Nouvel «avatar» de la modernité numérique, le hackathon s'invite de plus en dans les entreprises en matière d'innovation. Selon BemyApp [BEM 19], on enregistre 5636 hackathons publics et internes en 2018 dans le monde, soit près de $40 \%$ de plus qu'en 2016 . Un constat identique que fait Hackathon.com, la plus grande communauté de hackathon en ligne au monde.

A l'heure actuelle, les hackathons ne se cantonnent plus aux populations de développeurs. En 2018, 64\% des hackathons étaient ouverts au grand public [BEM 19]. L'ambition partagée par les entreprises est de conduire leur processus d'innovation de manière différente en se frottant à un écosystème d'acteurs à compétences variées, plus large que leurs collaborateurs. En ce sens, le

\footnotetext{
${ }^{18}$ JavaOne est une conférence annuelle organisée d'abord en 1996 par Sun Microsystems pour discuter de Java technologies, principalement parmi les développeurs Java. https://fr.qwerty.wiki/wiki/JavaOne 
hackathon est souvent considéré comme une première entrée en matière relativement simple d'Open Innovation.

L'émergence de cette pratique est sans doute aussi liée à son impact polymorphe dans l'approche d'innover. En effet, en dehors de la production de projets d'innovation, un hackathon peut porter plusieurs ambitions telles que: l'accélération de la transformation digitale d'une entreprise ; l'instauration d'une culture d'innovation et un fort degré d'implication des collaborateurs considéré comme une réelle pratique de team building ${ }^{19}$ - au sein de l'entreprise, notamment en favorisant l'entrepreneuriat; le positionnement en tant que marque innovante ; la fidélisation des clients et la fédération d'une communauté autour d'une marque orientée vers l'innovation, etc.

\subsubsection{Quelques cas emblématiques de pratique de Hackathon}

Compte tenu du nombre de hackathons dans le monde la liste est bien trop longue. Etant vite propagés dans les entreprises du numérique, on comprend bien que depuis 2006, Facebook soit en tête en termes de hackathons réalisés [BEL 18]. Le réseau social en organise très régulièrement pour ses employés ${ }^{20}$. Pour une entreprise classée dans le top 10 des entreprises les plus innovantes ${ }^{21}$, il n'est pas étonnant que le réseau social soit également connu pour valoriser la culture du « hacking » [UFF 17].

Dans un article du blog Facebook en 2012, Pedram Keyani, ancien directeur de l'ingénierie chez Facebook, révèle que de nombreuses fonctionnalités phares du réseau social, comme le «like » ou la « timeline » sont nées lors des hackathons. En effet, l'ancien directeur déclare :

«Certains de nos produits les plus aimés ont commencé lors de hackathons, ont eu des accessoires lors du forum prototype, et se sont rendus sur vos ordinateurs et téléphones, y compris la vidéo, le bouton J'aime, le chat, le hip-hop pour PHP et même la timeline. » ${ }^{22}$ (Pedram Keyani, "Stay focused and keep hacking”, 23 mai 2012 à 19h22)

Toutefois, Facebook est loin d'être le seul ténor à avoir organisé des hackathons. Avant lui, «Yahoo!» organisait en interne ses «Hackdays » depuis 2005. Google ou Apple en organisent aussi souvent pour leurs employés. Cette tradition est ancienne dans la Silicon Valley, de ce fait, on comprend parfaitement que les Etats-Unis soient en tête du classement avec 1511 hackathons enregistrés en 2018 [BEM 19].

\subsection{La place de la créativité collaborative dans les Hackathons}

\subsubsection{Le Hackathon: une méthodologie co-créative et agile}

Le terme anglo-saxon «hack », cœur de l'approche du Hackathon, renvoie à un esprit ludique, à la curiosité, à la ténacité et à la créativité. Pour rappel, le Hackathon est « un moment de compétition, où les participants travaillent en équipe sur une période déterminée et courte pour réfléchir, collaborer, concevoir, rapidement prototyper, tester, itérer et proposer des solutions créatives et innovantes » [MOB 19].

Le caractère collaboratif et limité dans le temps correspond le mieux aux premières étapes du processus d'innovation imprégné d'une combinaison de l'approche Design Thinking, de la

\footnotetext{
${ }^{19}$ En français renforcement d'équipe

${ }^{20}$ Ils font une apparition dans le célèbre film à succès « The Social Network » produit par David Fincher en 2010.

${ }^{21}$ Classement Forbes en 2018. https://www.forbes.fr/classements/entreprises/classement-forbes-des-entreprises-les-plusinnovantes-au-monde/?cn-reloaded=1

22 https://www.facebook.com/notes/facebook-engineering/stay-focused-and-keep-hacking/10150842676418920

(c) 2020 ISTE OpenScience - Published by ISTE Ltd. London, UK - openscience.fr 
méthodologie agile et du Lean Startup ${ }^{23}$. Cette méthodologie co-créative et agile du hackathon, qui est une conséquence intuitive du caractère imprévisible du marché, encourage le prototypage rapide et le test de plus d'idées possibles, afin de les transformer en des véritables projets d'innovation.

Le Design Thinking permet d'avoir une approche centrée sur l'homme pour résoudre de manière créative et globale des problèmes complexes à la suite de multiples itérations et implication d'acteurs et actifs diverses [BRO 08]. Le Lean start-up, quant à lui permet d'accélérer le processus de développement des solutions innovantes par une approche de production accélérée d'un «Produit Minimum Valable » qui est rapidement testé et validé par les utilisateurs.

Le hackathon est aujourd'hui utilisé dès qu'il s'agit de mettre en œuvre l'intelligence collective. Cette pratique dite «agile» encourage la créativité et l'expérimentation rapide [BRI 14]. En outre, souvent orientée défi, la pratique du hackathon enclenche la réalisation d'une démarche participative de résolution créative de problème qui augmente les probabilités de succès des projets d'innovation.

La méthodologie mise en place lors d'un hackathon doit permettre aux équipes de bénéficier de tout l'accompagnement nécessaire lors du développement de leur projet: de l'idéation à la structuration d'un projet clair, créatif et innovant. Ainsi, le hackathon s'inscrit dans des perspectives de co-conception, d'économie du partage et d'économie collaborative [RIC 15].

\subsubsection{L'organisation et l'animation d'un Hackathon}

De manière générale, pour parvenir à un véritable moment intense de résolution créative de problème en un délai très court, le principe du hackathon est simple : faire travailler des équipes pendant 12 à 72 heures, dans le but de développer des nouveaux concepts et les prototyper [MOB 19]. Pour arriver à des telles fins, tout hackathon doit être soigneusement planifié, exécuté et suivi. Le déroulé du hackathon, de manière générique, se fait en 3 phases : organisation, exécution et suivi.

La phase d'organisation concerne principalement la définition des paramètres techniques et stratégiques, à savoir l'identification des enjeux et la formulation des défis, la conception logistique du hackathon, le type d'intervenants (animateur, coach, jury), etc. Tous ces paramètres doivent être en adéquation avec les objectifs fixés, la culture d'entreprise, les ressources mises à disposition et le profil des participants.

La phase d'exécution concerne principalement l'animation du hackathon et l'accompagnement au développement des solutions. Elle emprunte toutes les phases de l'approche Design Thinking, qui permet d'appréhender un hackathon tel un véritable apprentissage organisé autour d'une problématique difficile et complexe qui nécessite de la collaboration, multiple réflexion et des compétences collectives [LAR 16].

De manière générale, un hackathon générique est organisé sous un format de deux jours ayant pour principales étapes : créer de l'empathie (Empathize), définir la problématique (Define), générer des solutions (Ideate), tester et prototyper (Iterate) et le Pitch.

\footnotetext{
${ }^{23}$ Méthode agile qui permet de réduire les cycles de conception des nouveaux produits. Elle se base sur la construction rapide d'un « Produit Minimum Valable » qui sera testé et validé par les utilisateurs pilotes. 


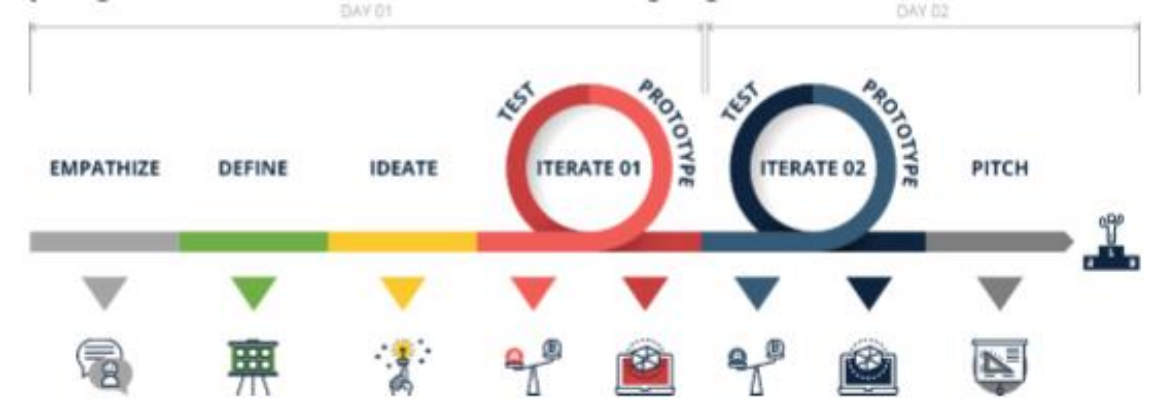

Figure 1. Processus de développement de projet pour un hackathon en format 2 jours [GUT 19]

La phase de suivi renvoi au processus d'implémentation des projets innovants portés par les lauréats du hackathon, celle-ci correspond le plus souvent à des programmes d'incubation proposés par les organisateurs. Il faut absolument que les projets qui émergent puissent être commercialisés pour être concrétiser en tant qu'innovations.

\subsubsection{Effet de levier sur la créativité collaborative}

Bien qu'il n'existe pas d'approche miracle pour impulser une dynamique d'innovation, les hackathons figurent comme une pratique permettant de libérer la créativité des collaborateurs et renforcer la collaboration avec tout l'écosystème. Cette pratique constitue une occasion idoine pour mobiliser l'intelligence collective en réponse à un défi formulé. Elle met en lumière des actifs (idées, connaissances, concepts, technologies, compétences, etc.) parfois inexploitées - en raison du silotage de l'organisation - et des échanges intenses et transverses qui contribuent à révéler le potentiel créatif.

Pour réussir à stimuler la créativité au sein de l'organisation, le hackathon s'appuie généralement, en plus de sa méthodologie co-créative et agile, sur l'approche évènementielle et la gamification [MOB 19]. L'approche évènementielle rend possible la mobilisation et la participation d'un grand nombre d'acteurs provenant de milieux divers. La gamification, quant à elle, par transposition partielle ou totale des enjeux d'une entreprise en une approche ludique et dans un esprit de compétition, permet de booster le processus de résolution des enjeux en insufflant aux participants une dynamique de challenge, qui déclenche le recours à un fort degré de créativité provoquée au cours du processus de développement des solutions nouvelles.

Le hackathon, s'appuyant sur une méthodologie co-créative et agile, couplé à l'approche évènementielle et à la gamification, tire donc sa particularité et performance dans sa capacité à développer le potentiel créatif de tous les participants [BLU 17], pour finalement les accompagner dans une démarche participative et co-créative de résolution de problèmes. Cette démarche de cocréation permet de susciter une réelle émulation de la part de tous, in fine, favorisant l'expression du potentiel créatif de toute l'organisation.

\section{Discussion critique sur la pratique du Hackathon}

\subsection{Le Hackathon: une pratique différente du crowdsourcing}

A l'heure actuelle, il n'est plus utile de débattre sur l'importance de l'ouverture d'une entreprise vers son écosystème. En outre, on constate que d'autres pratiques de recours au public, dans le but 
d'engendrer des concepts nouveaux ou améliorés, ont largement été intégrées par les organisations sous forme de stratégie de crowdsourcing $^{24}$.

Le crowdsourcing d'idéation ${ }^{25}$ permet au public de répondre à un problème posé par l'entité organisationnelle [MAJ 15]. Cette production participative de concepts novateurs est ouverte à tous, voire même que la participation des individus n'est soumise à aucun critère particulier. Par conséquent, plusieurs inconvénients peuvent être abordés, les plus importants sont ceux liés à la non-maitrise de la qualité des projets proposés, corollaire d'une absence de méthodologie structurée et orientée vers l'atteinte des objectifs de l'entreprise en quête d'innovations [REN 17].

Un proverbe africain dit « Tout seul on va plus vite, ensemble on va plus loin ». Dans une logique organisationnelle, cela suppose qu'il faudrait créer une forme de tension entre l'action individuelle et le résultat collectif. Ce risque est fort probable lorsqu'il s'agit principalement d'une pratique de crowdsourcing. Cette mise sous tension nécessite de s'appuyer sur la créativité collaborative pour garantir l'implication et la collaboration des acteurs divers dans le processus de résolution créative de problème. C'est pourquoi, nous considérons que le hackathon représente une pratique d'Open innovation plus aboutie que le crowdsourcing.

Comme susmentionné, à la différence du crowdsourcing, le hackathon tire sa particularité et sa performance dans la mobilisation de la créativité collaborative. De surcroit, cette pratique ne fait pas que la stimuler, en s’appuyant sur une méthodologie co-créative et agile (voir \$2.2.1.), elle permet une certaine maitrise de la qualité des projets proposés en alignement avec les objectifs de l'entreprise en quête d'innovation.

Le hackathon constitue dès lors une pratique ad hoc, qui déclenche le processus organisationnel durant lequel la créativité des individus suit des phases itératives et séquentielles à la suite de la mobilisation de l'intelligence collective. Le hackathon s'appuie donc sur la créativité collaborative pour générer le plus de concepts nouveaux ou améliorés possibles grâce à l'émulation provoquée, et surtout facilite l'adoption des solutions inédites développées au sein d'une organisation - par le truchement de sa méthodologie co-créative et agile. Ainsi, l'approche du hackathon constitue un levier de mobilisation de la créativité collaborative sans que cette dernière étouffe la créativité individuelle au sein de l'organisation.

\subsection{Le Hackathon : source de développement des futures innovations}

Bien que nombreuses définitions de l'innovation soient simplistes, en peu de mots, il s'agit du processus de création de valeur ${ }^{26}$ à partir d'idées et concepts nouveaux. Elle représente la capacité à soutenir et gérer un flux de nouveaux produits, processus et services au sein de toute organisation. L'innovation n'est pas un accident, ni un phénomène qui tombe du ciel à chaque instant, c'est généralement le résultat de la créativité appliquée [GOL 17].

Le hackathon pour sa part, en s'appuyant sur la créativité collaborative, représente un bon levier de développement du potentiel créatif d'une organisation. Cependant, la spirale d'innovation n'est complète que lorsque l'expression du potentiel créatif (concept nouveau) de l'organisation est introduite dans le circuit économique (marché ou entreprise). C'est pour dire que sans orientation et

\footnotetext{
${ }^{24}$ Le crowdsourcing ou la production participative, a été initialement défini comme le fait d'externaliser une tâche, habituellement réservée aux employés d'une firme, vers un large groupe d'individus extérieurs à l'entreprise sous la forme d'un appel ouvert à la participation de volontaires (Howe, 2006).

${ }^{25}$ C'est un type spécifique de production participative, qui fait appel à un grand nombre de personnes, généralement des internautes (Renard, 2017).

${ }^{26}$ La valeur créée est analysée alors à deux niveaux : valeur économique suite à la commercialisation sur le marché et gain de productivité pour l'entreprise suite à son intégration au sein de son organisation. 
engagement stratégiques, la créativité pourrait bien faire surface naturellement ou encore de manière provoquée au sein de toute entreprise, mais l'innovation ne sera pas forcément livrée [JOL 03].

Heureusement, l'efficacité du hackathon ne se limite pas qu'à la génération de concepts nouveaux, elle va plus loin jusqu'à déclencher la motivation et l'implication des participants dans le processus de co-création des solutions inédites pour des problèmes clairement identifiés ou non. Rappelons que lors d'un hackathon, il s'agit de réunir, pendant 12 à 72 heures, des professionnels d'horizons divers pour tenter «d'apporter de façon collaborative et ouverte des solutions originales et pratiques, généralement de nature technologique, à des problèmes qui restaient alors sans solution. Cette approche tend même aujourd'hui à s'élargir à des solutions sociotechniques ou organisationnelles, comme la conception de nouveaux processus » [DIO 16, P. 62]. En transformant le processus d'innovation par une approche co-créative, la méthodologie agile du hackathon imprégné du Design Thinking et du Lean startup - permet d'insuffler une forte dynamique d'innovation [BRO 09].

De tout ce qui précède, les hackathons se dévoilent comme un moyen de promotion et de renforcement de l'innovation économique, sociale et/ou intellectuelle [GRE 18]. Cette pratique cocréative - en s'appuyant sur la créativité collaborative - permet d'emprunter une démarche efficace de Design Thinking et Lean Startup. Au sens strict, elle enrichit les étapes d'idéation par la génération et maturation d'un plus grand nombre d'idées; elle stimule les itérations - plus particulièrement, par une accélération des phases de prototypage et de test - dont les résultats, généralement acceptés de tous, sont issus d'une perception à $360^{\circ 27}$; et enfin, elle aboutit à la conception des solutions innovantes inédites pour des problématiques qui tardaient à être résolues.

\section{Conclusion}

Les exigences d'innovation ordinaire à tous les niveaux de l'organisation et les évolutions de la compétition montrent les limites d'une approche de fermeture. Elles obligent les entreprises à rompre avec la tradition classique d'innovation, celle de passer des mois de recherche et des longues périodes à développer et tester des produits sur le marché, uniquement suivant un processus interne et cloisonné. Pour ce faire, elles invitent les entreprises à développer des organisations agiles et collaboratives capables de s'appuyer sur leur écosystème pour accéder à des ressources nouvelles afin d'innover plus vite, plus souvent...bref, innover de manière créative.

Dans ce contexte, le hackathon se révèle comme une pratique efficace d'entrée en matière pour l'Open innovation. Le hackathon représente un processus créatif et collaboratif qui implique la participation et l'implication de plusieurs acteurs de divers métiers [BRO 08]. Cette pratique trouve sa pertinence en raison de la réalité selon laquelle l'innovation ne doit pas être le seul apanage des ingénieurs, mais résulter d'un «bricolage » collectif. Ainsi, la créativité collaborative permet à l'organisation de mobiliser l'expression de son potentiel créatif, enrichit par l'implication de son écosystème, dans la génération des concepts nouveaux qui seront transformés en réelles innovations par l'entreprise.

En conclusion, comme déjà mentionné, le hackathon est une pratique d'innovation orientée vers la résolution de problèmes et centrée sur l'humain qui articule la pensée analytique et la pensée intuitive [BRO 09], Autrement dit, grâce à une méthodologie structurée stimulant la créativité de toute une organisation, le hackathon s'appuie sur la créativité collaborative pour aboutir au

\footnotetext{
${ }^{27}$ Le hackathon permet un rassemblement de différents acteurs de l'écosystème à compétences et expériences variées. II permet d'avoir une maitrise de tous les aspects du projet lors des phases de génération et maturation des solutions, et enfin celles du développement technique (prototypage et test).
} 
développement des concepts nouveaux et parfois inédits. Ces concepts nouveaux, développés à l'issue du hackathon - par le levier de la créativité collaborative - posent les bases des futures innovations des entreprises.

\section{Références}

[AGO 16] AGOGUE M., «Alex F. Osborn - Le brainstorming : le premier outil de support de la créativité », dans Cohendet P., Hussier C., Burger-Helmechen T., (dir), Les Grands Auteurs en Management de l'Innovation et de la Créativité, Editions EMS, Cormelles-le-Royal, 2016.

[AMA 96] AMABILE T. M., Creativity in context, Westview Press, Colorado, Etats-Unis, 1996.

[AMA 02] AMABILE T. M., HADLEY C., KRAMER S., «Creativity Under the Gun », Harvard Business Review, 80(2), 3-11, 2002.

[AMA 12] AMABILE T. M., Componential Theory of Creativity, Working Paper, 2012-04-26, Harvard Business School, 2012.

[AND 14] ANDERSON N., POTOČNIK K., ZHOU J., «Innovation and Creativity in Organizations: A State-of-theScience Review, Prospective Commentary, and Guiding Framework », Journal of Management, 40 (5), 1297 1333, 2014.

[BAR 88] BARRON F., Putting creativity to work, dans Stenberg R.J. (Ed.), The Nature of Creativity: Contemporary Psychological Perspectives, Cambridge University Press, Cambridge, 77-98, 1988.

[BEL 18] BELLIN I., «Les Hackathons font désormais partie de la stratégie d'innovation », Data Analytics Post, https://dataanalyticspost.com/les-hackathons-font-desormais-partie-de-la-strategie-dinnovation/ [accédé le 20 Mai 2019], 2018.

[BEM 19] BEMYAPP, «Infographic: Worldwide Hackathon Figures in 2018 and trends to expect in 2019 », https://www.bemyapp.com/insights/infographics-hackathon-figures-in-2018.html/ [accédé le 14 Aout 2019], 2019.

[BES 15] BESANÇON M., LUBART T., «Psychologie de la créativité », Encyclopaedia Universalis, http://www.universalis-edu.com/encyclopedie/psychologie-de-la-creativite/ [accédé le 25 Octobre 2019], 2015.

[BLU 17] BLUENOVE, «Gamifier pour mieux régner : la gamification comme levier de la créativité des organisations », https://bluenove.com/blog/gamifier-pour-mieux-regner/ [accédé le 20 Avril 2019], 2017.

[BOU 04] BOUILLERCE B., CARRE E., Savoir développer sa créativité, Retz, Paris, 2004.

[BOC 17] BOCCARA G., « Focus sur... les Hackathons : l'essentiel à savoir sur ce phénomène-clé », Welcome to the Jungle, https://www.welcometothejungle.com/fr/articles/focus-hackathons-essentiel-a-savoir [accédé le 20 Aout 2019], 2017.

[BRI 14] BRISCOE G., MULligAN C., Digital Innovation: The Hackathon Phenomenon, Creativeworks London, Working paper no.6, 2014-05, https://www.creativeworkslondon.org.uk/wp-content/uploads/2013/11/DigitalInnovation-The-Hackathon-Phenomenon1.pdf [accédé le 20 Aout 2019], 2014.

[BRO 08] BROWN T., « Design thinking », Harvard Business Review, 84-92, 2008.

[BRO 09] BROWN T., Change by Design: How Design Thinking Transforms Organizations and Inspires Innovation, Harper Collins, New York, 2009.

[CAM 11] CAMILLE C., GELINAS S., Créativité et gestion : Les idées au service de l'innovation, Presse de l'université du Québec, Canada, Québec, 2011.

[CAR 07] CARRIER C., «Cultiver la créativité et gérer l'innovation dans la PME », dans Filion, L.-J. (dir.), Management des PME, De la création à la croissance, Pearson Éducation, Paris, 119-130, 2007.

[CHO 07] CHOUTEAU M., VIEVARD L., «L'innovation, un processus à décrypter », dans Millénaire, le Centre Ressources Perspectives du Grand Lyon, p.24, 2007.

[CHE 03] CHESBROUGH H., Open Innovation: The new imperative for creating and profiting from technology? Harvard Business School Press, Boston, Massachusetts, 2003.

[CHE 06] CHESBROUGH H., Open Innovation: Researching a New Paradigm, Oxford University Press, Oxford, UK, 2006. 
[COH 16] COHENDET P., HUSSLER C., BURGER-HELMCHEN T., Les grands auteurs en management de l'innovation et de la créativité, Editions EMS, Cormelles-le-Royal, 2016.

[DES 08] DE BONO E., Serious Creativity: Using the Power of Lateral Thinking to Create New Ideas, HarperBusiness, 1992.

[DES 08] DE SOUSA F.C., "Still the elusive definition of creativity », International journal of psychology: a biopsychosocial approach, Vol.2, p. 55-82, 2008.

[DIO 16] DIONNE K.-E., CARLILE P., «Le pouvoir transformationnel des hackathons », dans Gestion: Revue Internationale de Gestion, 41, 62-63, 2016.

[FOR 14] FOREST J., « Petite histoire des modèles d'innovation », dans Boutillier S., Gallaud D., Forest J., Laperche B., Tanguy C., Temri L. (dir), Principes d'économie de l'innovation, Peter Lang, Bruxelles, 45-57, 2014.

[FOR 97] FOREST J., MICAËLLI J. P., PERRIN P., «Innovation et conception : Pourquoi une approche en termes de processus ? », 2ème Congrès International Franco-Québécois : Le Génie Industriel dans un monde sans frontière, Albi, Septembre, France, 1997.

[FER 10] FERNEZ-WALCH S., ROMON F., Management de l'innovation : de la stratégie aux projets, Vuibert, $2^{\text {ème }}$ Edition, Paris, 2010.

[GOL 17] GOLLER I., BESSANT J., Creativity for innovation management, London, Routledge, 2017.

[GOR 19] GORIA S., HUMBERT P., ROUSSEI B., Information, connaissance et créativité agile, Editions ISTE, Série Smart Innovation, London, UK, 2019.

[GUT 19] GUTZMANN M., «Using hackathons to accelerate procurement's digital transformation », Smartprocurement,

http://www.smartprocurement.co.za/using hackathons to accelerate procurements digital transformation.php\#st hash.jyQG4GNY.3ZXZ4fAI.dpbs/ [accédé le 14 Octobre 2019], 2019.

[GRE 18] GRESELLE-ZAÏBET O., KLEBER A., DEJOUX C., « Le hackathon en mode Design Thinking ou quelles modalités pour former à des compétences méthodologiques et comportementales ?» Management \& Avenir, 104,149-171, 2018.

[HER 95] HERAUD J. A., « Brevets et contexte institutionnel de la création technologique », dans, Baslé M., et al. (dir.), Changements institutionnel et changement technologique, CNRS Éditions, Lonrai, 91-117, 1995.

[HER 99] HERMAN N., Creatitivity? Innovation ? Are They Different ? Do We Need Both ? FOCUS, 10(3), maijuin, 1999.

[HOW 06] HOWE, J., The rise of crowdsourcing, Wired magazine, 14(6), 1-4, 2006.

[IRI 07] IRISH CENTER FOR DESIGN INNOVATION, The design difference. A survey and design and innovation amongst Ireland's SME's Sligo, Ballinode. http://www.designinnovation.ie/downloads/TheDesignDiffer-ence 2007.pdf [accédé le 14 Octobre 2019], 2007.

[JOL 03] JOLY A., Innovation: Harnessing Creativity for Business growth, Kogan Page Publishers, London, 2003.

[KLI 86] KLINE S., ROSENBERG N., An overwiew of innovation, Landau R., Rosenberg N. (eds.), The Positive Sum strategy, 275-305, 1986.

[KOM 15] KOMSSI M., PICHLIS D., RAATIKAINEN M., KINDSTRÖM K., JÄRVINEN J., « What are hackathons for? », IEEE SOFTWARE, 32(5), 60-67, 2015.

[LAM 17] LAMBERT DESJARLAIS M. P., MICHEL E., POIRIER A., VANDEN-ABEELE L., "Créativité et innovation », dans Gardoni M., Navarre A. (dir), Pratique de gestion de l'innovation : Guide sur les strategies et les processus, Québec, Presses de l'université du Québec, 7-28, 2017.

[LAR 16] LARA M., LOCKWOOD K., «Hackathons as Community-Based Learning: a Case Study », TechTrends, Washington DC, 60(5), 486-495, 2016.

[LUB 94] LUBART T., « Creativity », dans Sternberg R.J. (Ed.), Handbook of Perception and Cognition: Thinking and problem solving, New York, Academic Press, 298-332, 1994.

[LUB 15] LUBART T., MOUCHIROUD C., SYLVIE T., ZENASNI F., Psychologie de la créativité, Armand Colin, $2^{\text {ème }}$ Edition, Paris, 2015. 
[LUB 19] LUBART T., BESANÇON M., BARBOT B., « Creativity, a potential resource for children and adolescents to evaluate, reveal and develop ", Neuropsychiatrie de l'Enfance et de l'Adolescence, 67(3), 121-129. https://www.sciencedirect.com/science/article/pii/S0222961719300285, 2019.

[MAC 62] MACKINNON D.W., « The nature and nurture of creative talent », American Psychologist, 17(7), 484495, 1962.

[MAJ 15] MAJCHRZAK A., MALHOTRA A., « Towards an information systems perspective and research agenda on crowdsourcing for innovation », Journal of Strategic Information Systems, 22(4), 257-268, 2013.

[MOB 19] MOBHE BOKOKO D., Développement de l'innovation participative : l'effet de la pratique des Hackathons sur l'accélération du processus d'innovation des entreprises, Mémoire de Master 2 Management de l'innovation, Université du Littoral Cote d'Opale, 2019.

[MOO 93] MOORE J. F., «Predators and Prey; A New Ecology of Competition », Harvard Business Review, MayJune, 75-86, 1993.

[MOO 96] MOORE J. F., The Death of Competition. Leadership \& Strategy in the Age Business Ecosystem, HaperBusiness, New York, 1996.

[OCH 90] OCHSE, R., Before the gates of excellence: The determinants of creative genius, Cambridge University Press, Cambridge, 1990.

[OSB 53] OSBORN A., Applied imagination : Principles and Procedures of Creative Problem Solving, Charles Scribner's Sons, New York, 1953.

[PEN 16] PENIN J., «Joseph Aloïs Schumpeter - Père de l'économie et de la gestion de l'innovation », dans Cohendet P., Hussier C., Burger-Helmechen T., (dir), Les Grands Auteurs en Management de l'Innovation et de la Créativité, Editions EMS, Cormelles-le-Royal, 2016.

[REN 17] RENARD D., «La production participative d'idées: quells effets du dispositive socio-technique sur les pratiques? » dans Baillargeon D., Coutant A. (dir), Créativité et organisations: Tensions communicationnelles, Communiquer, Revue de communication sociale et publique, 21, 63-78, 2017.

[RIC 15] RICHARDSON L., « Performing the sharing economy », Geoforum, 67, 121-129, 2015.

[RUN 12] RUNCO M.A., JAEGGER G. J., « The Torrance Creativity Center. The Standard Definition of Creativity », Creativity Research Journal, 24(1), 92-96, 2012.

[SAM 07] SAMIER H., SANDOVAL V., La Webcréativité, Hermes science, Lavoisier, Collection finance \& gestion \& management, Paris, 2007.

[STER 95] STERNBERG R. J., LUBART T., Defying the crowd: cultivating creativity in a culture of conformity, Free Press, New York, 1995.

[TEG 18] TEGLBORG A.-C., GLASER A., «Les événements collaboratifs, vecteurs de l'innovation participative dans trois grandes entreprises », Entreprendre \& Innover, 3(38), 9-16, 2018.

[TET 09] TETHER B., Design in Innovation Coming out from the Shadow of R\&D. An Analysis of the UK Innovation Survey of 2005, Department for Innovation, Universities and Skills. HM Government, London, 2009.

[UFF 17] UFFREDUZZI M., Hackathon as Emerging Innovation Practice: Exploring Opportunities and Challenges through 8 in-depth Case Studies, Politecnico di Milano, Milano, https://www.politesi.polimi.it/bitstream/10589/137237/5/Hackathon\%20as\%20Emerging\%20Innovation\%20Practi ce.pdf [accédé le 20 Avril 2019], 2017.

[WA 26] WALLAS G., The art of thought, Harcourt, Brace and Company, New York, 1926. 